\title{
Editorial
}

\section{Sensor/Actuator Networks and Networked Control Systems}

\author{
Ge Guo, ${ }^{1}$ Xin Wang, ${ }^{2}$ Linying Xiang, ${ }^{3}$ and Fuzhong Nian ${ }^{4}$ \\ ${ }^{1}$ School of Information Science and Technology, Dalian Maritime University, Dalian 116026, China \\ ${ }^{2}$ School of Electronic, Information and Electrical Engineering, Shanghai Jiaotong University, Shanghai 200240, China \\ ${ }^{3}$ Department of Automation, Xiamen University, Xiamen 361005, China \\ ${ }^{4}$ School of Computer and Communication, Lanzhou University of Technology, Lanzhou 730080, China
}

Correspondence should be addressed to Ge Guo; geguo@yeah.net

Received 12 August 2014; Accepted 12 August 2014; Published 27 August 2014

Copyright (C) $2014 \mathrm{Ge}$ Guo et al. This is an open access article distributed under the Creative Commons Attribution License, which permits unrestricted use, distribution, and reproduction in any medium, provided the original work is properly cited.

\section{Introduction}

The last two decades have witnessed a great deal of interests in sensor/actuator networks (SANs), or, generally, networked control systems (NCSs). Compared to conventional system architectures, NCSs have numerous advantages such as reduced system wiring, being easier for design, diagnosis, and maintenance, lower cost, and increased flexibility, reliability, and safety. As a result, NCSs have been widely applied to many areas, for example, automobiles, aircrafts, and spacecrafts, autonomous vehicles, transportation systems, power systems, remote monitoring and data acquisition systems, chemical processes, and many manufacturing plants.

Research on NCSs has heretofore focused mainly on several basic communication constraints that can cause performance degradation or stability loss, such as network-induced delays, packet dropouts, data corruptions or disordering, and data rate limitation and quantization effects. Medium access constraint is another important issue worthy of intensive investigation, which refers to the fact that the network cannot accommodate all the nodes (sensors, actuators, subsystems, etc.) simultaneously at any time. There are other open issues awaiting investigation, too.

As a special type of NCSs, sensor/actuators networks typically have a large number of devices advanced in sensing, communicational, computational, and mobility capabilities, demanding nontrivial energy. In addition, the batteries powering these devices have limited capacity and cannot be replaced or recharged conveniently. As a consequence, many interesting and challenging issues are open, for example, localization, coverage and routing of mobile sensor/actuator networks, and so forth.

The special issue focuses on the state-of-the-art research and development in the analysis and design for networked control systems as well as the theoretical and technological advances in sensor and actuator networks. The intention of this special issue was to provide for researchers a forum to share their latest research results on filtering, estimation, control, and other aspects in sensor/actuator networks and networked control systems and for readers an opportunity to have an overview of the latest achievements about some interesting issues this field has been encountering.

In the next section, we give a brief description of the papers in this special issue.

\section{An Overview of the Special Issue}

This special issue comprises twenty-six papers, which are carefully selected from many submissions by a rigorous peer review process. Roughly, the articles in this special issue can be classified into three topics: control and estimation of NCSs, corporative control and filtering of multiagent systems, and theoretical and practical issues in sensor and networks.

Falling within the topic area of control and estimation of networked systems are seven papers, dealing with stabilization or estimation problems of systems subject to constraints like delay, dropout, and quantization, including 
applications in various industrial fields. To name some, the paper entitled " $H_{\infty}$ guaranteed cost control for networked control systems under scheduling policy based on predicted error," by Q. Zhu et al., studies scheduling policy based on model prediction errors with the aim of reducing energy consumption and network conflicts at the actuator node. The actuator nodes are assumed to have limited energy and high collision probability and the sensor nodes are responsible for state prediction based on a model. The transmission control strategy is an essentially event-triggered one; that is, the control signal is transmitted only when the prediction error violates a threshold set by the controller. The design method of the guaranteed cost controller is presented by considering parameter uncertainty and long-time delay.

Another interesting paper on this topic is "Control and optimization of network in networked control system," by Z. Wang and H. Sun, which investigates the relationship between quality of performance (QoP) of control systems and quality of service (QoS) of the communication network. It presents an idea to avoid network congestion from the view point of control theory, which marks the departure of this work from the existing results. Specifically, the congestion and bandwidth are regarded as the state and control variables, respectively. Using these variables, a linear time-invariant system model is established to describe the connection between the congestion state and the network bandwidth. A linear quadratic method is introduced to eliminate network congestion by allocating bandwidth dynamically.

On the other hand, a couple of papers focus on the state estimation and filtering problems in NCSs or SANs. In "Distributed $H_{\infty}$ sampled-data filtering over sensor networks with Markovian switching topologies," by B. Yang et al., a distributed sampled-data filtering problem is studied for sensor networks with stochastic switching topologies and transmission delay. The network topology switching is triggered by a Markov chain. A distributed filter structure is given, in which each sensor has access to the measurement of its neighbor nodes. The distributed sampled-data filtering problem is transformed into the stability problem of a Markovian jump error system. Then, in the context of mean-square stability analysis and using Lyapunov-Krasovskii method, a topologydependent sampled-data filtering method is obtained.

Along this line, there is another paper "Varianceconstrained robust estimation for discrete-time systems with communication constraints," by B. Wang et al., which is concerned with the filtering problem in NCSs subject to measurement quantization, random transmission delay, and packets loss. The aim of this paper is to design a linear filter such that, for all the communication constraints, the error state of the filter is mean-square bounded and the steady-state variance of the estimation error of each state variable is no larger than a prescribed bound.

Another topic of this special issue regards theoretical developments in multiagent systems, with the emphasis on consensus control and consensus filtering problems. There are in total four papers on this topic, focusing, respectively, on latest advances in cooperative filtering, control and tracking of multiagent systems, and application to an extrusion machine producing process. In "Consensus tracking of multiagent systems with time-varying reference state and exogenous disturbances," by $\mathrm{H}$. Yang et al., tracking control of multiagent systems is studied. A path following algorithm with a time-varying reference state is proposed, and the path tracking performance of multiagent systems with exogenous disturbances is analyzed. A disturbance observerbased control method is developed, which can guarantee asymptotical consensus of multiagent systems under either fixed or switching topologies, regardless of the time-varying reference state and the exogenous disturbance.

Dr. Y. Liu et al. contributed a noteworthy work "Distributed Kalman-consensus filtering for sparse signal estimation," which presents a Kalman filtering-based distributed algorithm for sparse signal estimation. The authors suggested a rebuilt pseudomeasurement-embedded Kalman filter in the form of information set and an improved parameter selection approach. By introducing the pseudomeasurement technique into the Kalman-consensus filtering problem, a distributed estimation algorithm is developed, which fuses the measurements from different nodes in the network and hence renders all filters to reach a consensus on the estimate of the sparse signals.

Furthermore, we have seven papers studying a variety of theoretic issues in sensor and actuator networks (including general complex networks) and eight papers discussing various applications. To save space, here, we just mention a few of them. For instance, in "Energy efficient low-cost virtual backbone construction for optimal routing in wireless sensor networks," by K. M. Pitchai and B. Paramasivan, an efficient weighted connected dominating sets (CDS) algorithm is presented for constructing a low-cost virtual backbone with hop spanning ratio and minimum number of dominators. The approach has three phases, with the initial phase revoking a partial CDS tree from a complete CDS tree and the second and final phases giving the CDS algorithm by determining the dominators using an iteration process.

$\mathrm{K}$. Zhao et al. proposed in "Cooperative transmission in mobile wireless sensor networks with multiple carrier frequency offsets: a double-differential approach" a relay selectionbased double-differential cooperative transmission scheme, in which the best relay sensor node is selected to forward the source sensor node's signals to the destination node with the detect-and-forward (DetF) protocol. Assuming a Rayleigh fading environment, the closed-form expressions for the outage probability and average bit error rate of the scheme are first derived. Then, expressions for the asymptotic outage probability and the average bit error rate for large signal-to-noise ratio (SNR) are presented. Finally, a simple analytical solution to the optimal power allocation problem is obtained by minimizing the average bit error rate.

As transmission security issue is very important, in "Applying 3D polygonal mesh watermarking for transmission security protection through sensor networks," Dr. R. Hu et al. discussed the problem of copyright protection and digital right management. In this paper, a blind watermarking algorithm is proposed for security protection in transmitting $3 \mathrm{D}$ polygonal meshes through sensor networks. The presented method is based on selecting prominent feature vertices (prongs) on the mesh and then embedding the same 
watermark into their neighborhood regions. The embedding algorithm is based on modifying the distribution of vertex norms by using quadratic programming. Decoding results are obtained by a majority voting scheme over neighborhood regions of the prongs. In situations where cropping cannot remove all prongs, robustness against the cropping attack can be achieved both theoretically and experimentally, showing that the results provide a solution for $3 \mathrm{D}$ polygonal watermarking potential to withstand a variety of attacks.

\section{Concluding Remarks}

Due to space limitation, we cannot introduce all the papers in this special issue one by one with more details. However, we do hope that this special issue contains useful information that can help motivate more researchers to contribute to this fascinating area.

\section{Acknowledgments}

We appreciate all reviewers who dedicated their time and effort to perform review of all the submitted papers and provided valuable suggestions to the authors.

Ge Guo

Xin Wang

Linying Xiang

Fuzhong Nian 


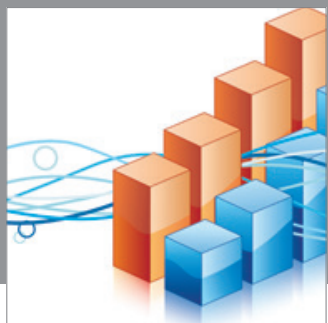

Advances in

Operations Research

mansans

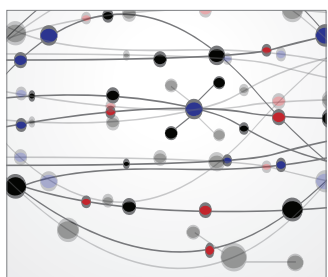

The Scientific World Journal
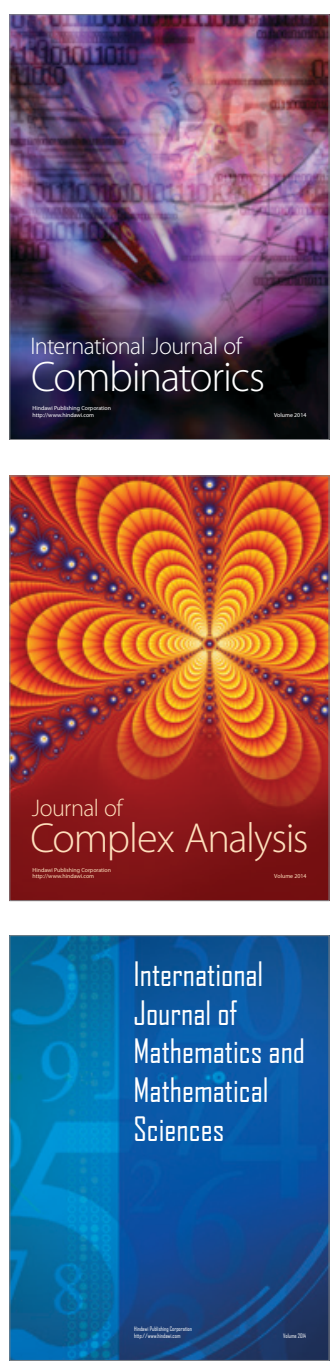
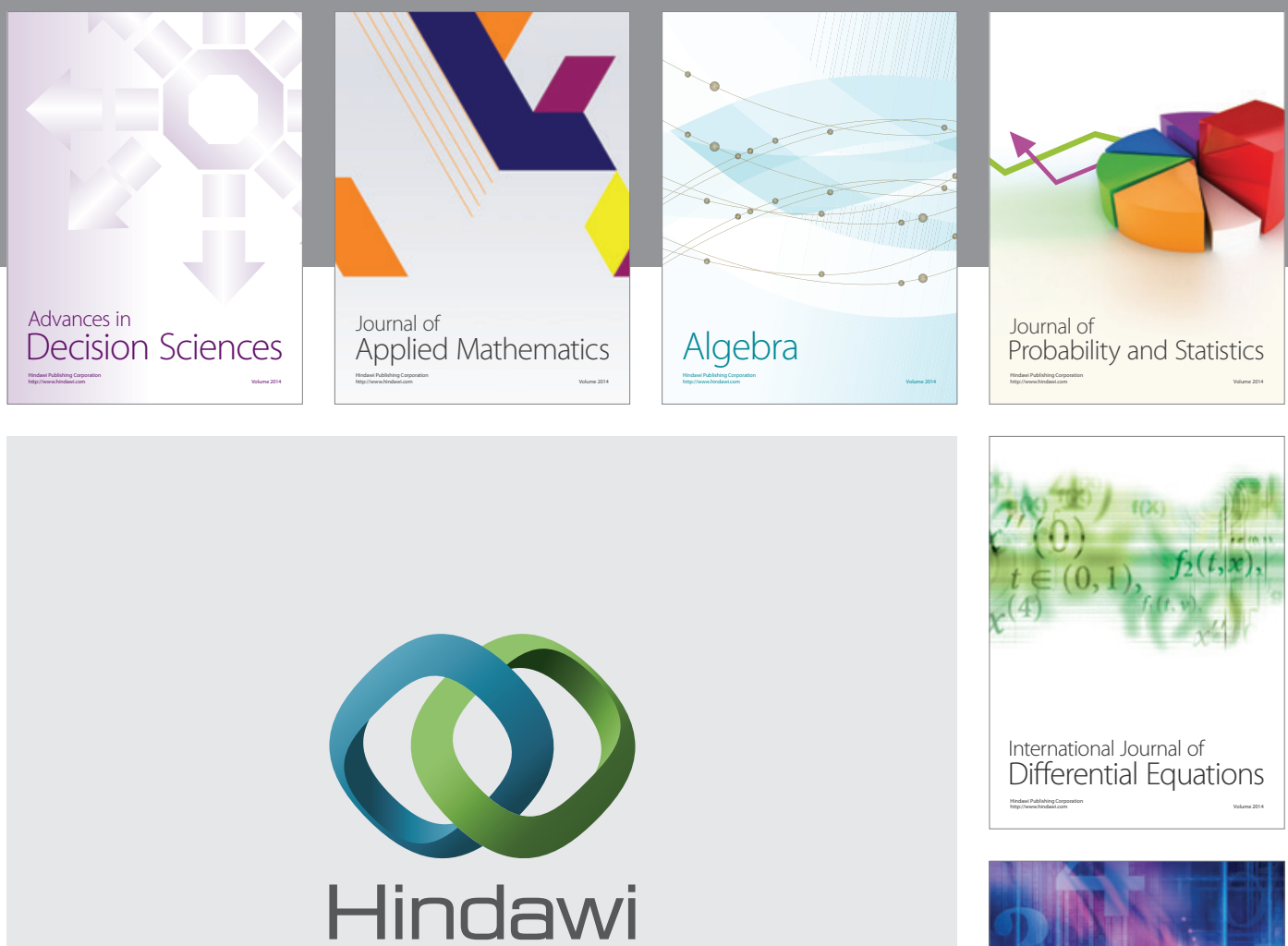

Submit your manuscripts at http://www.hindawi.com
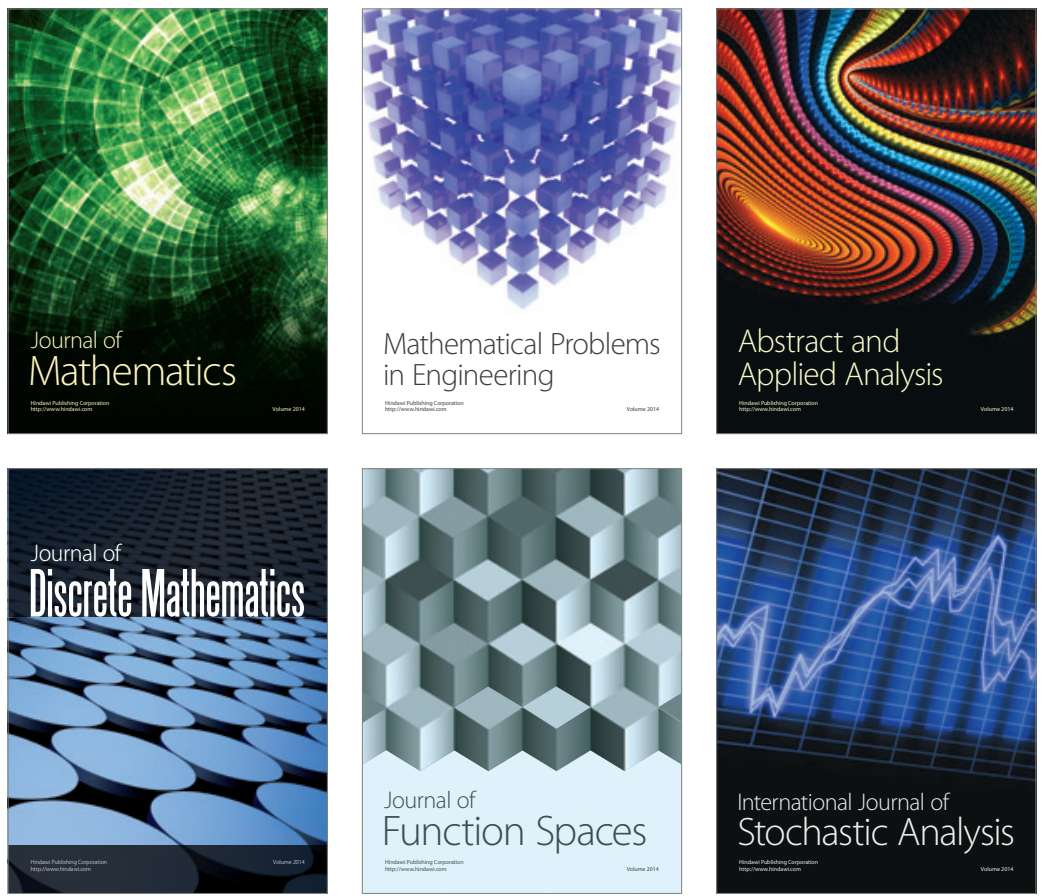

Journal of

Function Spaces

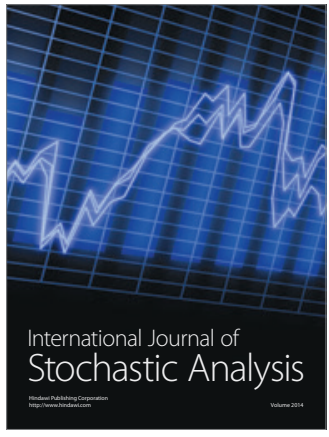

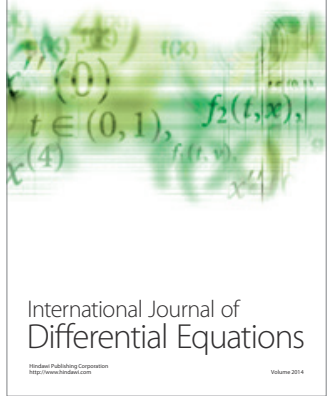
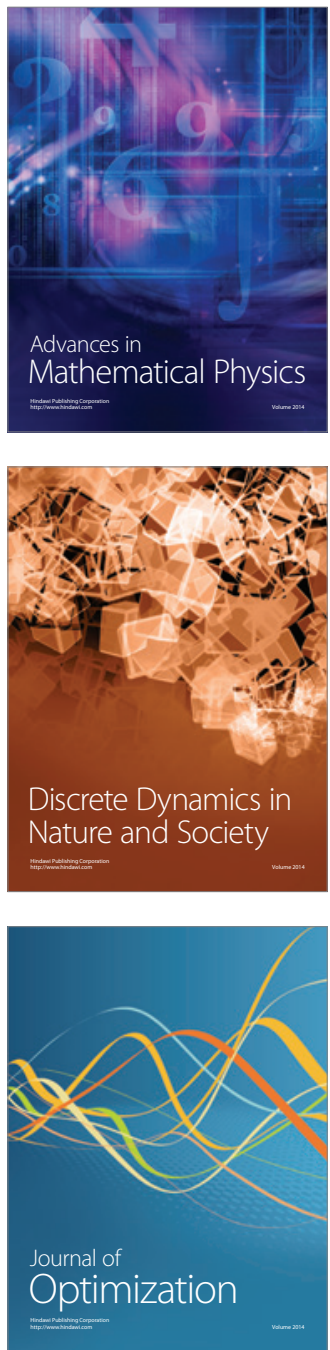